\title{
Connection between asymptotic normalization coefficients, subthreshold bound states, and resonances
}

\author{
A. M. Mukhamedzhanov and R. E. Tribble \\ Cyclotron Institute, Texas A\&M University, College Station, Texas 77843
}

(Received 14 September 1998)

\begin{abstract}
We present here useful relations showing the connection between the asymptotic normalization coefficient (ANC) and the fitting parameters in $K$ - and $R$-matrix theory methods which are often used when analyzing low energy experimental data. It is shown that the ANC of a subthreshold bound state defines the normalization of both direct radiative capture leading to this state and resonance capture in which the state behaves like a subthreshold resonance. A determination of the appropriate $\mathrm{ANC}(\mathrm{s})$ thus offers an alternative method for finding the strength of these types of capture reactions, both of which are important in nuclear astrophysics. [S0556-2813(99)01006-7]

PACS number(s): 24.30.-v, 25.40.Lw, 26.30.+k
\end{abstract}

\section{INTRODUCTION}

Nuclear excited states below the particle emission threshold typically undergo $\gamma$ decay to lower lying states. These decays result in the initial states having their own natural width. In the case when $\gamma$ emission is the only open decay channel, the natural width $\Gamma_{\gamma}$ is typically $\sim e V$. If a particle bound excited state lies very close to the particle threshold, the natural width can result in the tail of the wave function extending above the particle threshold. As a result of this tail, the subthreshold bound state can behave like a resonance state in a capture reaction. Such states are often referred to as subthreshold resonance states [1] and they can play an important role in determining reaction rates of interest in nuclear astrophysics.

Consider the capture of particle $b$ by particle $a$ at very low relative kinetic energy $E$ and assume that there is a subthreshold bound state $c 1$ in the system $c=(a b)$. There are three possible mechanisms by which the capture can occur [1]: (i) direct radiative capture to the ground state $c$, (ii) radiative capture to the ground state through the subthreshold resonance, and (iii) direct radiative capture into the subthreshold bound state with $\gamma$ emission.

Process (ii) corresponds to nonradiative capture of particle $b$ into the subthreshold resonance $c 1$. The excited state then undergoes $\gamma$ decay to the ground state $c$. The energy of the emitted photon is

$$
E_{\gamma}=E+\varepsilon_{c},
$$

where $\varepsilon_{c}$ is the binding energy of the ground state $c=(a b)$. Note that only one gamma is emitted in the process and it occurs after capture into the $c 1$ state. Process (iii) results initially in a photon with energy

$$
E_{\gamma}=E+\varepsilon_{c 1} .
$$

The subthreshold bound state $c 1$ is then deexcited to the ground state $c$ by emitting a photon with energy $\varepsilon_{c}-\varepsilon_{c 1}$. Note that in mechanisms (ii) and (iii) the capture occurs into the same state, but in (ii) this state reveals itself as a resonance, while in (iii) it acts as a real bound state. All three of these capture processes occur in nature and are important in determining reaction rates for nuclear astrophysics.

In previous papers [2-5] we have pointed out that the overall normalization of the cross section for a direct radiative capture reaction at low binding energy is entirely defined by the asymptotic normalization coefficient (ANC) of the final bound state wave function into the two-body channel corresponding to the colliding particles. Below we show how to extend this to capture into subthreshold resonance states. Typically the approaches used to analyze low energy experimental data in order to derive astrophysical factors are the $K$ and $R$-matrix methods. We will present equations relating the ANC to the residue of the pole corresponding to the subthreshold bound state in the $K$-matrix method and the reduced width amplitude in the $R$-matrix method. In the case of a Breit-Wigner-type resonance (above threshold), the ANC is related to the resonance width. The equations given here have direct experimental implications and can be used in the analysis of experimental data. When analyzing data using the $K$ - or $R$-matrix methods, the parameters corresponding to the subthreshold bound states can be fixed by measuring ANC's independently from transfer reactions $[4,5]$. Also by measuring ANC's one can simultaneously determine astrophysical factors both for direct radiative capture to the subthreshold bound state and for capture to the subthreshold resonance. The equations presented below are correct for scattering amplitudes in $K$ - and $R$-matrix theory at negative energies, and so they can be used to find the ANC by extrapolating elastic scattering data (phase shifts) to the pole corresponding to the subthreshold bound state [6].

In what follows we use the system of units in which $\hbar$ $=c=1$.

\section{ASYMPTOTIC NORMALIZATION COEFFICIENT}

We present first some useful equations for the ANC. Let us consider a virtual decay of nucleus $c$ into two nuclei $a$ and $b$. First we introduce the overlap function $I$ of the bound state wave functions of particles $c, a$, and $b$ : 


$$
\begin{aligned}
I_{a b}^{c}(\mathbf{r})= & \left\langle\varphi_{a}\left(\zeta_{a}\right) \varphi_{b}\left(\zeta_{b}\right) \mid \varphi_{c}\left(\zeta_{a}, \zeta_{b} ; \mathbf{r}\right)\right\rangle \\
= & \sum_{l_{c} m_{l_{c}} j_{c} m_{j_{c}}}\left\langle J_{a} M_{a} j_{c} m_{j_{c}} \mid J_{c} M_{c}\right\rangle \\
& \times\left\langle J_{b} M_{b} l_{c} m_{l_{c}} \mid j_{c} m_{j_{c}}\right\rangle i^{l_{c}} Y_{l_{c} m_{l_{c}}}(\hat{\mathbf{r}}) I_{a, b l_{c} j_{c}}^{c}(r),
\end{aligned}
$$

where for each nucleus $\varphi$ is the bound state wave function, $\zeta$ are a set of internal coordinates including spin-isospin variables, and $J$ and $M$ are the spin and spin projection. Also $\mathbf{r}$ is the relative coordinate of the centers of mass of nuclei $a$ and $b, \hat{\mathbf{r}}=\mathbf{r} / r, j_{c}, m_{j_{c}}$ are the total angular momentum of particle $b$ and its projection in the nucleus $c=(a b), l_{c}, m_{l_{c}}$ are the orbital angular momentum of the relative motion of particles $a$ and $b$ in the bound state $c=(a b)$ and its projection, $\left\langle j_{1} m_{1} j_{2} m_{2} \mid j_{3} m_{3}\right\rangle$ is a Clebsch-Gordan coefficient, $Y_{l_{c} m_{c}}(\hat{\mathbf{r}})$ is a spherical harmonic, and $I_{a b, l_{c} j_{c}}^{c}(r)$ is the radial overlap function which includes the antisymmetrization factor due to identical nucleons. The summation over $l_{c}$ and $j_{c}$ is carried out over the values allowed by angular momentum and parity conservation in the virtual process $c \rightarrow a+b$. Since the radial overlap function is not a solution of the Schrödinger equation, it is approximated by a model wave function of the bound state $c=(a b)$ as follows:

$$
I_{a b l_{c} j_{c}}^{c}(r)=S_{a b l_{c} j_{c}}^{1 / 2} \varphi_{n_{c} l_{c} j_{c}}(r) .
$$

Here $\varphi_{n_{c} l_{c} j_{c}}(r)$ is the bound state wave function for the relative motion of $a$ and $b$ which can be calculated, for example, in the shell model or resonating group method and is normalized by

$$
\int_{0}^{\infty} d r r^{2} \varphi_{n_{c} l_{c} j_{c}}^{2}(r)=1
$$

$S_{a b l_{c} j_{c}}$ is the spectroscopic factor of the configuration $(a b)$ with quantum numbers $l_{c}, j_{c}$ in nucleus $c$. It is defined as the norm of the radial overlap function $[7,4]$

$$
S_{a b l_{c} j_{c}}=\int_{0}^{\infty} d r r^{2}\left[I_{a b l_{c} j_{c}}^{c}(r)\right]^{2} \text {. }
$$

The asymptotic normalization coefficient $C_{a b l_{c} j_{c}}^{c}$ defining the amplitude of the tail of the radial overlap function $I_{a b l_{c} j_{c}}^{c}(r)[7,4]$ is given by

$$
I_{a b l_{c} j_{c}}^{c}(r) \stackrel{r>R_{N}}{\rightarrow} C_{a b l_{c} j_{c}}^{c} \frac{W_{-\eta_{c}, l_{c}+1 / 2}\left(2 \kappa_{a b} r\right)}{r},
$$

where $R_{N}$ is the nuclear interaction radius between $a$ and $b$, $W_{-\eta_{c}, l_{c}+1 / 2}\left(2 \kappa_{c} r\right)$ is the Whittaker function describing the asymptotic behavior of the bound state wave function of two charged particles, $\kappa_{c}=\sqrt{2 \mu_{a b} \varepsilon_{c}}$ is the wave number of the bound state $c=(a b), \mu_{a b}$ is the reduced mass of particles $a$ and $b$, and $\eta_{\kappa_{c}}=Z_{a} Z_{b} \mu_{a b} / \kappa_{c}$ is the Coulomb parameter of the bound state $(a b)$. The ANC is related to the nuclear vertex constant $G_{a b l_{c} j_{c}}^{c}$ by $[7,2]$

$$
G_{a b l_{c} j_{c}}^{c}=-\exp \left[i \pi\left(\frac{l_{c}+\eta_{c}}{2}\right)\right] \frac{\sqrt{\pi}}{\mu_{\alpha}} C_{a b l_{c} j_{c}}^{c} .
$$

Taking into account the asymptotic behavior of the bound state wave function

$$
\varphi_{n_{c} l_{c} j_{c}}(r) \stackrel{r>R_{N}}{\rightarrow} b_{l_{c} j_{c}} \frac{W_{-\eta_{c}, l_{c+1 / 2}}\left(2 \kappa_{c} r\right)}{r},
$$

where $b_{l_{c} j_{c}}$ is the single-particle ANC defining the amplitude of the tail of the bound state wave function at large $r$, we easily derive, from Eqs. (4), (7), and (9),

$$
\left(C_{a b l_{c} j_{c}}^{c}\right)^{2}=S_{a b l_{c} j_{c}} b_{l_{c} j_{c}}^{2}
$$

The ANC is related to the residue of the elastic scattering amplitude in the so-called direct pole in the energy plane corresponding to the bound state. To show this we introduce the transition matrix $\mathcal{T}$, which is related to the $S$ matrix as

$$
S=1-\mathcal{T}
$$

The diagonal partial $S$-matrix element is given by $S_{j j}$ $=\exp \left(2 i \delta_{l}\right)$, where $\delta_{l}$ is the full scattering phase shift in the partial wave $l$ which includes the Coulomb scattering phase shift $\sigma_{l}$ also. Thus in our approach the $S$ and $\mathcal{T}$ matrices include the Coulomb phase shift if it is nonzero. Note that usually the Coulomb rescattering is singled out - i.e., only the Coulomb-modified nuclear phase shift is considered but we take into account the total scattering phase shift. Let us consider now the elastic scattering $a+b \rightarrow a+b$. Let $j$ stand for the channel $a+b$. If $a$ and $b$ can form the bound state $c=(a b)$ with binding energy $\varepsilon_{c}$ and relative orbital angular momentum $l$ (for simplicity we omit the subscript $c$ in $l$ ), then the elastic scattering amplitude has a pole corresponding to this bound state in the $l$ th partial wave at the relative kinetic energy of particles $a$ and $b, E=-\varepsilon_{c}$. In the momentum plane it corresponds to the pole at $k=i \kappa_{c}$, where $E=k^{2} / 2 \mu_{a b}$ with $k$ being the relative momentum of particles $a$ and $b$. Near this pole the partial elastic transition amplitude $T_{j j}$ in the $l$ th partial wave can be written in the form [8]

$$
T_{j j}(k) \stackrel{k \rightarrow i \kappa_{c}}{\approx}(-1)^{l} i e^{i \pi \eta_{\kappa_{c}}} \frac{|C|^{2}}{k-i \kappa_{c}} .
$$

Thus the ANC simultaneously defines the normalization of the tail of the overlap function and the residue in the pole corresponding to the bound state of the partial elastic transition amplitude. This connection follows from the particle conservation law in nonrelativistic quantum mechanics [8].

\section{III. $K$-MATRIX APPROACH AND THE ANC}

\section{A. Relating the ANC to the pole residue for the subthreshold bound state and the resonance width}

Consider the radiative capture process

$$
a+b \rightarrow c+\gamma,
$$

where the final nucleus $c$ has an excited bound state which is very close to the threshold for $a+b$. For convenience, we 
assume that the constituent particles $a$ and $b$ in channel $c$ are spinless. We also assume that there are no close resonances at low relative kinetic energy $E$ between particles $a$ and $b$. Then we need to take into account only two channels $j$ and $\gamma$, which correspond to channels $a+b$ and $c+\gamma$, respectively. The transition matrix has two-components, $T_{j j}$ which corresponds to the elastic scattering $a+b \rightarrow a+b$ and $T_{\gamma j}$ which corresponds to the radiative capture (13) to the ground state through the subthreshold resonance. For simplicity we consider only two bound states in the system $(a b)$, the ground state and the excited subthreshold bound state. Since $T_{\gamma j}$ is significantly smaller than $T_{j j}$, one can write

$$
\begin{gathered}
T_{\gamma j}=2 i p_{\gamma} p_{j} \frac{K_{\gamma j}}{1+i \mu_{j} K_{j j}}, \\
T_{j j}=2 i p_{j}^{2} \frac{K_{j j}}{1+i \mu_{j} K_{j j}} .
\end{gathered}
$$

The diagonal elements $p_{j}, p_{\gamma}$, and $\mu_{j}$ of the diagonal matrices $p$ and $\mu$ in channels $j$ and $\gamma$ are given by

$$
\begin{gathered}
p_{j}=e^{-(\pi \eta / 2) \operatorname{sgn} \operatorname{Re} k} \frac{\Gamma(l+i \eta+1)}{l !} k^{l+1 / 2}, \\
\mu_{j}=\left|p_{j}\right|^{2}, \\
p_{\gamma}=k_{\gamma}^{l \gamma^{+1 / 2}} .
\end{gathered}
$$

Here $\eta=Z_{a} Z_{b} \mu_{a b} / k$ is the Coulomb parameter, $l$ is the relative orbital angular momentum of particles $a$ and $b$ in channel $j, k_{\gamma}$ is the momentum of the photon emitted during the transition from the subthreshold bound state $c$ to the ground state, and $l_{\gamma}$ is its multipolarity. Since we consider only $\operatorname{Re} k>0$, even when extrapolating to the bound state pole $k$ $=\lim \operatorname{Re} \mathrm{k} \rightarrow+0+i \operatorname{Im} k$, we can take $\operatorname{sgn} \operatorname{Re} k=1$.

Let us consider the partial element $T_{j j}$ in the partial wave $l$ where particles $a$ and $b$ form the subthreshold bound state $c 1$. The excited bound state close to threshold has a width caused by its $\gamma$ transition to lower lying bound states. At low relative energies $E$ in channel $j$, the subthreshold bound state can be "seen" by the incident particle $b$; i.e., it can be captured into the subthreshold bound state of nucleus $c$ as a resonance state with subsequent $\gamma$ transition to the bound state. The matrix element describing the capture to the subthreshold resonance is given by Eq. (14).

For certain classes of local nuclear potentials, the $K$ matrix is a real symmetric matrix. Moreover, the matrix elements of the $K$ matrix are analytic functions of $k^{2}$ at $k^{2}=0$ with a branch cut on part of the negative real axis and with isolated poles on the cut in the complex $k^{2}$ plane $[9,10]$. Since the matrix elements $K_{j j}$ and $K_{\gamma j}$ are meromorphic functions of $k^{2}$ except for the cut, we present them in the Padé form

$$
K_{\gamma j}=\frac{P_{N_{1}}}{Q_{M}}, \quad K_{j j}=\frac{D_{N_{2}}}{Q_{M}},
$$

where $P_{N}, D_{N}, Q_{N}$ are polynomials of $N$ th order in the $k^{2}$ plane. Consider first the transition matrix element $T_{j j}$. Taking into account the Padé parametrization of $K_{j j}$, we get

$$
T_{j j}=2 i p_{j}^{2} \frac{D_{N_{2}}}{Q_{M}+i \mu_{j} D_{N_{2}}} .
$$

As has been indicated, the elastic transition matrix element $T_{j j}$ has a pole at $k=i \kappa_{c 1}$ where $\kappa_{c 1}=\sqrt{2 \mu_{a b} \varepsilon_{c 1}}$ is the wave number corresponding to the subthreshold bound state $c 1$. $T_{j j}$ also has a pole corresponding to the ground state of $c$ but we do not consider it as we assume that it is quite far from the subthreshold bound state. We now show how to relate the residue of $T_{j j}$ in the pole corresponding to the subthreshold bound state to the ANC. To do this, we must extrapolate $T_{j j}$ to the bound state pole located on the physical sheet of the $k$ plane at $k=i \kappa_{c 1}$, i.e., to the positive imaginary axis in the complex $k$ plane or to the negative real axis in the $E$ plane. Since $\mu_{j}$ is a modulus of $p_{j}^{2}$, it is not an analytic function, and when extrapolated down to negative energies, $\mu_{j}=0$ at $E \leqslant 0$. However, Eq. (16) shows that $p_{j}^{2}$ is an analytic function in the $k$ plane. If we write

$$
p_{j}^{2}=e^{2 i \sigma_{l}} \mu_{j},
$$

then it becomes clear why the Coulomb scattering, given by $\exp \left(2 i \sigma_{l}\right)$, was included in $T_{j j}$ since without this factor, $p_{j}^{2}$ would not be analytic and its extrapolation to negative energies would lead to the wrong residue (see the Appendix). Thus at $E<0$ we get

$$
T_{j j}=2 i p_{j}^{2} \frac{D_{N_{2}}}{Q_{M}} .
$$

Hence the pole of $T_{j j}$ at negative energy corresponds to the zero of $Q_{M}$. It is convenient to represent the ratio $D_{N_{2}} / Q_{M}$ as a sum of pole terms plus a background $B_{j}$ :

$$
\frac{D_{N_{2}}}{Q_{M}}=\sum_{\lambda=1}^{M} \frac{g_{c \lambda}^{2}}{k^{2}-k_{\lambda}^{2}}+B_{j} .
$$

Then

$$
T_{j j}{ }^{2}<0<i p_{j}^{2} \sum_{\lambda=1}^{M} \frac{g_{c \lambda}^{2}}{k^{2}-k_{\lambda}^{2}}+B_{j},
$$

where $g_{c \lambda}^{2}$ is the pole residue. Note that some but not all of the poles in the expansion (24) correspond to bound states in $c$ [9]. Let $\lambda=1$ correspond to the subthreshold bound state $c 1$. Then, at $k^{2} \rightarrow k_{1}^{2}=-\kappa_{c 1}^{2}$,

$$
T_{j j} \stackrel{k^{2} \rightarrow-\kappa_{c 1}^{2}}{\approx} 2 i p_{j}^{2} \frac{g_{c 1}^{2}}{k^{2}+\kappa_{c 1}^{2}} .
$$

Recall that the elastic transition amplitude $T_{j j}$ near the pole corresponding to the bound state was given by Eq. (12). Comparing Eqs. (25) and (12) we find the relationship between $g_{c 1}$ and the ANC. For $k \rightarrow i \kappa_{c 1}$,

$$
p_{j}=e^{i \pi \eta_{\kappa_{c 1}} / 2} \frac{\Gamma\left(l+\eta_{\kappa_{c 1}}+1\right)}{l !}\left(i \kappa_{c 1}\right)^{l+1 / 2} .
$$

Hence 


$$
T_{j j} \stackrel{k^{2} \rightarrow-\kappa_{c 1}^{2}}{\approx} i(-1)^{l} e^{i \pi \eta_{\kappa_{c 1}}}\left[\frac{\Gamma\left(l+\eta_{\kappa_{c 1}}+1\right)}{l !}\right]^{2} \kappa_{c 1}^{2 l} \frac{g_{c 1}^{2}}{k-i \kappa_{c 1}},
$$

with the expression for $g_{c 1}$,

$$
g_{c 1}^{2}=\frac{1}{\kappa_{c 1}^{2 l}} \frac{(l !)^{2}}{\Gamma^{2}\left(l+1+\eta_{\kappa_{c 1}}\right)}|C|^{2},
$$

following from Eqs. (12) and (27). Thus the residue of the closest pole of $K_{j j}$ is proportional to the corresponding ANC. Although we assumed that particles $a$ and $b$ are spinless, Eq. (28) is valid also for particles with nonzero spins. Allowing for spin, the ANC and the residue $g_{c 1}$ also depend on the total angular momentum $j_{c 1}$ of particle $b$ in the bound state (in $j j$ coupling) or on the spin channel (in $L S$ coupling).

Consider now $T_{\gamma j}$. From (19) we immediately arrive at

$$
T_{\gamma j}=2 i p_{\gamma} p_{j} \frac{1}{Q_{M} / P_{N_{1}}+i \mu_{j}\left(D_{N_{2}} / P_{N_{1}}\right)} .
$$

Once again we introduce the pole expansion for

$$
K_{\gamma j}=P_{N_{1}} / Q_{M}=\sum_{\lambda=1}^{M} \frac{g_{\gamma \lambda} g_{c \lambda}}{k^{2}-k_{\lambda}^{2}}+B_{\gamma} .
$$

At small $\kappa_{c 1}^{2}$, where $k_{1}^{2}=-\kappa_{c 1}^{2}$, and $k^{2} \rightarrow 0$, we can use the one pole approximation giving

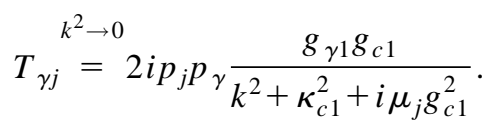

Comparing this equation with the Breit-Wigner amplitude, we find the relationship between the partial width $\Gamma_{c 1}$ of the subthreshold resonance seen by the incident particle $b$ at $E$ $>0$ and the residue $g_{c 1}^{2}$ in the $K$-matrix approach:

$$
\begin{aligned}
\Gamma_{c 1}(E) & =\frac{\mu_{j}}{\mu_{a b}} g_{c 1}^{2} \\
& =\frac{1}{\mu_{a b}}\left(\frac{k}{\kappa_{c 1}}\right)^{2 l} k e^{-\pi \eta}\left(\frac{|\Gamma(l+i \eta+1)|}{\Gamma\left(l+1+\eta_{\kappa_{c 1}}\right)}\right)^{2}|C|^{2},
\end{aligned}
$$

while the $\gamma$ width of the subthreshold resonance $\Gamma_{\gamma}$ and $g_{\gamma 1}$ are related by

$$
\Gamma_{\gamma}(E)=2 k_{\gamma 1}^{2 l l_{\gamma}+1} g_{\gamma 1}^{2}
$$

where $k_{\gamma}$ is the momentum of the photon emitted during the transition from the subthreshold bound state to the ground state $c$. The total width of the subthreshold resonance at positive energies is

$$
\Gamma(E)=\Gamma_{c 1}(E)+\Gamma_{\gamma}\left(E_{\gamma}\right) \approx \Gamma_{c 1}(E) .
$$

Thus the total width of the subthreshold resonance at $E>0$ is proportional to $|C|^{2}$.
We can now find the behavior of the cross section for capture to the subthreshold resonance at $E \rightarrow 0$. The cross section for this capture is given by

$$
\begin{aligned}
\sigma_{l \gamma}= & (2 l+1) \frac{2 \pi \mu_{a b}}{k^{2}}\left|T_{\gamma j}\right|^{2} \\
= & (2 l+1) \frac{\pi}{k^{2}} \frac{\Gamma_{\gamma} \Gamma_{c 1}}{\left(E+\varepsilon_{c 1}\right)^{2}+\Gamma_{c 1}^{2} / 4} \\
= & (2 l+1) \frac{\pi}{\mu_{a b} k}\left(\frac{k}{\kappa_{c 1}}\right)^{2 l} \\
& \times e^{-\pi \eta}\left(\frac{|\Gamma(l+i \eta+1)|}{\Gamma\left(l+1+\eta_{\kappa_{c 1}}\right)}\right)^{2} \\
& \times \frac{\Gamma_{\gamma}|C|^{2}}{\left(E+\varepsilon_{c 1}\right)^{2}+\Gamma_{c 1}^{2} / 4} \\
E & \approx 0 \\
\approx & (2 l+1) \frac{\pi^{2} \kappa_{c 1}}{\mu_{a b}^{2}} \frac{1}{E} e^{-2 \pi \eta} \\
& \times \frac{\left(\eta_{\kappa_{c 1}}\right)^{2 l+1}}{\Gamma^{2}\left(l+1+\eta_{\kappa_{c 1}}\right)} \frac{\Gamma_{\gamma}|C|^{2}}{\left(E+\varepsilon_{c 1}\right)^{2}} .
\end{aligned}
$$

Hence the astrophysical factor at $E \rightarrow 0$ behaves as

$$
\begin{aligned}
S(E)= & E e^{2 \pi \eta} \sigma_{l \gamma} \stackrel{E \rightarrow 0}{\approx}(2 l+1) \frac{\pi^{2} \kappa_{c 1}}{\mu_{a b}^{2}} \\
& \times \frac{\left(\eta_{\kappa_{c 1}}\right)^{2 l+1}}{\Gamma^{2}\left(l+1+\eta_{\kappa_{c 1}}\right)} \frac{\Gamma_{\gamma}|C|^{2}}{\left(E+\varepsilon_{c 1}\right)^{2}} .
\end{aligned}
$$

Thus we have shown that the ANC of the subthreshold bound state defines the overall normalization of the cross section and therefore the astrophysical factor for the capture into the subthreshold resonance at $E \rightarrow 0$. Usually when fitting low energy experimental data in the $K$-matrix approach, the one pole approximation is not sufficient. Nevertheless, the main fitting parameter $g_{c 1}$ can be fixed from an independent measurement of the ANC.

\section{B. Subthreshold bound state, ANC, and the scattering length}

Consider now the relationship between the ANC and the scattering length assuming that there is a subthreshold $s$-wave $(l=0)$ bound state $c 1$. The scattering amplitude is related to $T_{j j}$ by

$$
f_{j j}=-\frac{1}{2 i k} T_{j j}
$$

Consider now the behavior of $f_{j j}$ at $k \rightarrow 0$ : 


$$
\begin{aligned}
f_{j j} & \approx-0 \\
& \approx-e^{-\pi \eta} \Gamma^{2}(i \eta+1) \frac{K_{j j}}{1+\mu_{j} K_{j j}} \\
& \Gamma^{2}(i \eta+1) \frac{g_{c 1}^{2}}{k^{2}+\kappa_{c 1}^{2}+i \mu_{j} g_{c 1}^{2}} .
\end{aligned}
$$

We used in Eq. (41) the single-pole approximation for $K_{j j}$

$$
K_{j j} \stackrel{k \rightarrow 0}{\approx} \frac{g_{c 1}^{2}}{k^{2}+\kappa_{c 1}^{2}} .
$$

Approximation (42) is valid at $k^{2} \rightarrow 0$ and small enough $\kappa_{c 1}^{2}$. At $k \rightarrow 0$,

$$
f_{j j} \stackrel{k \rightarrow 0}{\approx}-e^{2 i \sigma_{0}^{C}} e^{-(\pi \eta)}|\Gamma(i \eta+1)|^{2} \frac{g_{c 1}^{2}}{\kappa_{c 1}^{2}} .
$$

The quantity

$$
\alpha=\frac{g_{c 1}^{2}}{\kappa_{c 1}^{2}}
$$

is nothing but the scattering length. Taking into account Eq. (28) we derive the relationship between the scattering length and the ANC:

$$
\alpha=\frac{1}{\kappa_{c 1}^{2}} \frac{1}{\Gamma^{2}\left(1+\eta_{\kappa_{c 1}}\right)}|C|^{2} .
$$

\section{IV. $R$-MATRIX APPROACH AND THE ANC}

Below we present some useful equations relating ANC's and paramerters in the $R$-matrix method. Although the $R$-matrix method was developed for analysis of resonance reactions, the reduced width of the $R$ matrix, which corresponds to the subthreshold resonance, can be related to ANC's of the subthreshold bound states. Let us consider the elastic scattering $a+b \rightarrow a+b$ at $k \rightarrow 0$ assuming the presence of the subthreshold bound state $c 1$. We note that the elastic scattering $S$-matrix element in channel $j$ is given by $[11,12]$

$$
S_{j j}=e^{-2 i\left(\phi_{l}-\sigma_{l}\right)} \frac{1 / R_{l}-\left[\Delta_{l}(E)-B_{l}-i \mathcal{V}_{l}(E)\right]}{1 / R_{l}-\left[\Delta_{l}(E)-B_{l}+i \mathcal{V}_{l}(E)\right]},
$$

where $R_{l}$ is the $R$ matrix for the $l$ th partial wave, $\Delta_{l}(E)$ is the Thomas shift, $B_{l}$ is the energy-independent $R$-matrix boundary condition constant, and $\mathcal{V}_{l}(E)$ is given by

$$
\mathcal{V}_{l}(E)=k r_{0} P_{l}(E) \text {. }
$$

$P_{l}(E)$ is the penetration factor which is given by

$$
P_{l}(E)=\frac{1}{G_{l}^{2}\left(k, r_{0}\right)+F_{l}^{2}\left(k, r_{0}\right)},
$$

where $r_{0}$ is the channel radius, and $G_{l}\left(k, r_{0}\right)$ and $F_{l}\left(k, r_{0}\right)$ are the singular (at the origin) and regular solutions of the radial Schrödinger equation with a pure Coulomb potential at $E>0$, i.e.

$$
e^{2 i \phi_{l}}=\frac{G_{l}\left(k, r_{0}\right)+i F_{l}\left(k, r_{0}\right)}{G_{l}\left(k, r_{0}\right)-i F_{l}\left(k, r_{0}\right)}
$$

The elastic scattering amplitude is given by the sum of two terms,

$$
\mathcal{T}_{j j}=1-S_{j j}=T_{j j}^{(\mathrm{pot})}+T_{j j},
$$

where $T_{j j}^{(\text {pot })}$ is the potential scattering amplitude and $T_{j j}$ is the so-called resonance scattering amplitude which is

$$
T_{j j}=-2 i e^{-2 i\left(\phi_{l}-\sigma_{l}\right)} \frac{\mathcal{V}_{l}(E)}{R_{l}-\left[\Delta_{l}(E)-B_{l}+i \mathcal{V}_{l}(E)\right]} .
$$

In the above equation, the $R$ matrix is

$$
R_{l}=\sum_{\lambda} \frac{\gamma_{c \lambda}^{2}}{E_{c \lambda}-E}
$$

where $E_{c \gamma}$ are the poles of the $R$ matrix and $\gamma_{c \lambda}$ is the reduced width of the $\lambda$ th level. If the energy of the subthreshold bound state is very close to threshold and the incident energy $E \rightarrow 0$, we can use the one-level $R$-matrix approximation which leads to

$$
T_{j j}=-2 i e^{-2 i\left(\phi_{l}-\sigma_{l}\right)} \frac{\mathcal{V}_{l}(E) \gamma_{c 1}^{2}}{E_{c 1}-E-\left[\Delta_{l}(E)-B_{l}+i \mathcal{V}_{l}(E)\right] \gamma_{c 1}^{2}}
$$

A priori the poles of the $R$ matrix do not coincide with the poles of the $S$ matrix. However, if we choose the boundary condition parameter $B_{l}=\Delta_{l}\left(-\epsilon_{c 1}\right)$, then the level shift of the subthreshold bound state disappears, $E_{c 1}=-\varepsilon_{c 1}$ [13], and Eq. (53) reduces to

$$
T_{j j}=-2 i e^{-2 i\left(\phi_{l}-\sigma_{l}\right)} \frac{\mathcal{V}_{l}(E) \tilde{\gamma}_{c 1}^{2}}{-\varepsilon_{c 1}-E-i \mathcal{V}_{l}(E) \tilde{\gamma}_{c 1}^{2}},
$$

where the effective reduced width of the subthreshold bound state is

$$
\tilde{\gamma}_{c 1}^{2}=\frac{\gamma_{c 1}^{2}}{1+\left.\gamma_{c 1}^{2}\left[d \Delta_{l}(E) / d E\right]\right|_{E=-\epsilon_{c 1}}} .
$$

Next we extrapolate Eq. (54) down to the bound state pole at $E=-\varepsilon_{c 1}$. The factor $\mathcal{V}_{l}(E)$ can be written as

$$
\mathcal{V}_{l}(E)=k r_{0} \frac{1}{\left|G_{l}\left(k, r_{0}\right)+i F_{l}\left(k, r_{0}\right)\right|^{2}} .
$$

This is not an analytic function. As we did when considering the $K$ matrix, we take $\mathcal{V}_{l}(E)=0$ at $E<0$ in the denominator, since $\mathcal{V}_{l}(E)$ is the imaginary part of the logarithmic derivative of the wave function which is real at negative energies. However, in the numerator we have 


$$
\begin{aligned}
e^{-2 i\left(\phi_{l}-\sigma_{l}\right)} \mathcal{V}_{l}(E) & =k r_{0} \frac{1}{e^{-2 i \sigma_{l}\left[G_{l}\left(k, r_{0}\right)+i F_{l}\left(k, r_{0}\right)\right]^{2}}} \\
& =k r_{0} \frac{1}{\left[u_{l}^{(+)}\left(k, r_{0}\right)\right]^{2}},
\end{aligned}
$$

where

$$
u_{l}^{(+)}\left(k, r_{0}\right)=(-i)^{l} e^{\pi \eta / 2} W_{-i \eta, l+1 / 2}\left(-i 2 k r_{0}\right)
$$

$u_{l}^{(+)}\left(k, r_{0}\right)$ is an analytic function in the entire complex plane, $|k|<\infty$, except at $k=0$ [14]. Hence we can extrapolate Eq. (54) down to the subthreshold bound state pole, bypassing the singular point $k=0$. At negative energies near this pole, we get

$$
T_{j j} \stackrel{k \rightarrow i \kappa_{c 1}}{\approx} 2 i \mu_{a b}(-1)^{l} e^{i \pi \eta_{\kappa_{c 1}}} \frac{r_{0}}{W_{-\eta_{\kappa_{c 1}}, l+1 / 2}^{2}\left(2 \kappa_{c 1} r_{0}\right)} \frac{\tilde{\gamma}_{c 1}^{2}}{k-i \kappa_{c 1}} .
$$

Comparing Eqs. (12) and (59) gives

$$
\tilde{\gamma}_{c 1}^{2}=\frac{1}{2 \mu_{a b}} \frac{W_{-\eta_{\kappa_{c 1}}, l+1 / 2}^{2}\left(2 \kappa_{c 1} r_{0}\right)}{r_{0}}|C|^{2} .
$$

From Eqs. (28) and (60) we find the relationship between the residue in the pole of the subthreshold bound state $c 1$ in the $K$-matrix method and $\tilde{\gamma}_{c 1}[13]$ :

$$
\frac{g_{c 1}^{2}}{2 \mu_{a b}}=\frac{1}{\kappa_{c 1}^{2 l}} \frac{(l !)^{2}}{\Gamma^{2}\left(l+1+\eta_{\kappa_{c 1}}\right)} \frac{r_{0}}{W_{-\eta_{\kappa_{c 1}}, l+1 / 2}^{2}\left(2 \kappa_{c 1} r_{0}\right)} \widetilde{\gamma}_{c 1}^{2} .
$$

The relationship between the ANC and the dimensionless effective reduced width amplitude $\widetilde{\theta}_{c 1}$ of the subthreshold bound state $c 1$ is

$$
\widetilde{\theta}_{c 1}^{2}=\mu_{a b} r_{0}^{2} \widetilde{\gamma}_{c 1}^{2}=\frac{r_{0}}{2} W_{-\eta_{\kappa_{c 1}}, l+1 / 2}\left(2 \kappa_{c 1} r_{0}\right)|C|^{2} .
$$

This result coincides with Eq. (16) in Ref. [15] and Eq. (7) in Ref. [16] if one takes into account that the normalization factor $N_{f}\left(N_{c}\right)$ in [15-17] is given by (for the one-channel case)

$$
N_{f}=\frac{1}{1+\left.\gamma_{c 1}^{2}\left[d \Delta_{l}(E) / d E\right]\right|_{E=-\epsilon_{c 1}}},
$$

and the dimensionless reduced width amplitude $\theta_{c l}^{2}$ $=2 \mu_{a b} r_{0}^{2} \gamma_{c 1}^{2}$. Note, however, that the ANC is model independent while the effective reduced width $\widetilde{\theta}_{c 1}$ depends on the channel radius $r_{0}$.

Having $\tilde{\gamma}_{c 1}$ expressed in terms of the ANC, the partial width of the subthreshold resonance $c 1$ at $E>0$ is given by

$$
\Gamma_{c 1}=2 \mathcal{V}_{l}(E) \tilde{\gamma}_{c 1}^{2}=\frac{1}{\mu_{a b}} \mathcal{V}_{l} \frac{W_{-\eta_{\kappa_{c 1}}, l+1 / 2}^{2}\left(2 \kappa_{c 1} r_{0}\right)}{r_{0}}|C|^{2}
$$

Thus the only model dependence of the subthreshold resonance partial width comes through the channel radius $r_{0}$.

Since the normalization of the radiative capture cross sections in cases (ii) and (iii) is defined by $g_{c 1}^{2} \sim|C|^{2}$, we have shown that in both cases the overall normalization of the cross section is defined by the same quantity — the ANC for the subthreshold bound state.

\section{ANC AND THE WIDTH OF THE ABOVE THRESHOLD RESONANCE}

We found above the relationship between the ANC and the width of the subthreshold resonance in the $K$ - and $R$-matrix methods. However, the ANC is also directly related to the width of a resonance which is above threshold. We will give this relationship for a Breit-Wigner resonance located at $E_{r}=E_{0}-i \Gamma / 2\left(\Gamma / E_{0}<1\right)$. The partial $S$-matrix element near the isolated Breit-Wigner resonance is given by [18]

$$
S_{j j}(k)=e^{2 i \nu_{l}} \frac{\left(k+k_{r}\right)\left(k-k_{r}^{*}\right)}{\left(k-k_{r}\right)\left(k+k_{r}^{*}\right)},
$$

where the partial scattering phase shift $\nu_{l}$ is a smooth function of energy near the resonance and real at real $k$ and $k_{r}$ $\approx k_{0}-i\left(\mu_{a b} / 2 k_{0}\right) \Gamma$. At $k \rightarrow k_{r}$,

$$
S_{j j}(k) \stackrel{k \rightarrow k_{r}}{=} \frac{A_{l}}{k-k_{r}}+g_{j j}
$$

where $g_{j j}$ is the regular function at $k=k_{r}$. The residue of the $S$ matrix in the resonance pole in leading order (up to terms of order $\approx \Gamma / 2 E_{0}$ ) is

$$
A_{l} \approx-i e^{2 i \nu_{l}\left(k_{0}\right)} \frac{\mu_{a b}}{k_{0}} \Gamma
$$

One can also use the Gamow wave function $\psi_{k_{r} l}(r)$, which is a regular solution of the radial Schrödinger equation describing the relative motion of particles $a$ and $b$ interacting via the sum of the nuclear and Coulomb potentials at resonance energy $E_{r}$, to describe the resonance. The asymptotic behavior of $\psi_{k_{r} l}(r)$ at large distances is

$$
\psi_{k_{r} l}(r) \stackrel{r \rightarrow \infty}{\approx} b_{l} \frac{e^{i\left(k_{r} r-\eta_{r} \ln 2 k_{r} r\right)}}{r} .
$$

Here $b_{l}$ is the single-particle ANC of the Gamow function and $\eta_{r}=Z_{a} Z_{b} \mu_{a b} / k_{r}$. Using the regularization procedure introduced by Baz', Zel'dovich, and Perelomov for resonance states [8], the Gamow wave function can be normalized to 1 by

$$
\lim _{\beta \rightarrow+0} \int_{0}^{\infty} d r r^{2} e^{-\beta r^{2}} \psi_{k_{r} l}^{2}(r)=1 .
$$


The relationship between the residue of the $S$ matrix in the resonance pole can also be written as [19]

$$
A_{l}=-i(-1)^{l} b_{l}^{2} \text {. }
$$

Comparing Eqs. (67) and (70) gives [19]

$$
b_{l}^{2} \approx(-1)^{l} e^{2 i \nu_{l}\left(k_{0}\right)} \frac{\mu_{a b}}{k_{0}} \Gamma_{s p} .
$$

It is important to note that Eq. (71) defines the relationship between the single-particle ANC, $b_{l}$, and the single-particle $\gamma$ width $\Gamma_{s p}$. Multiplying both sides of Eq. (71) by the spectroscopic factor $S_{l}$ we find

$$
C_{l}^{2} \approx(-1)^{l} e^{2 i \nu_{l}\left(k_{0}\right)} \frac{\mu_{a b}}{k_{0}} \Gamma
$$

\section{CONCLUSION}

We have presented equations relating the ANC of the subthreshold bound state with the residue of the $K$-matrix method in the pole corresponding to this bound state and with the reduced width in the $R$-matrix method. In the presence of the subthreshold bound state, there are two possible mechanisms of capture, direct capture to the subthreshold bound state and capture to the subthreshold resonance. It follows from Eqs. (28), (32), and (60) that the ANC of the subthreshold bound state defines the overall normalization of the cross sections and, hence, the astrophysical factors for both capture mechanisms. Thus by independently measuring the ANC for the subthreshold bound state, one can calculate the astrophysical factors for both capture mechanisms.

Several techniques are available to determine ANC's. One of the ways to extract them is to extrapolate the experimental phase shift to the pole of the elastic scattering amplitude corresponding to the subthreshold bound state [6]. Equation (27) gives the correct behavior of the elastic scattering amplitude at negative energies. Also we have shown $[4,5]$ that peripheral nucleon transfer reactions are a useful tool to extract ANC's for bound states. Equation (72) shows that transfer reactions to resonance states also can be used to extract directly the widths of the resonances.

\section{ACKNOWLEDGMENTS}

One of us (A.M.M.) would like to thank F.C. Barker for many useful discussions. This work was supported in part by the U.S. Department of Energy under Grant No. DE-FG0593ER40773.

\section{APPENDIX}

If we formally use $\mu_{j}=\left|p_{j}\right|^{2}$ instead of $p_{j}^{2}$ in Eq. (25) when extrapolating to the subthreshold bound state, we get different results for the residues in that bound state pole. Let us consider for simplicity $l=0$. At $E>0$ from Eq. (16) we derive

$$
\mu_{j}=\left|p_{j}\right|^{2}=\left|e^{-(\pi \eta / 2)} \Gamma(i \eta+1) k^{1 / 2}\right|^{2}=\frac{2 \pi \eta}{e^{2 \pi \eta}-1} k .
$$

Extrapolating Eq. (A1) to negative energies, we derive

$$
\mu_{j}=i \kappa_{c 1} e^{i \pi \eta_{\kappa_{c 1} 1}} \frac{\pi \eta_{\kappa_{c 1}}}{\sin \left(\pi \eta_{\kappa_{c 1}}\right)}
$$

However, the extrapolation of $p_{j}^{2}$ to negative energies gives

$$
p_{j}^{2}=i \kappa_{c 1} e^{i \pi \eta_{\kappa_{c 1}} \Gamma^{2}\left(\eta_{\kappa_{c 1}}+1\right) .}
$$

[1] C.E. Rolfs and W.S. Rodney, Cauldrons in the Cosmos (The University of Chicago Press, Chicago, 1988).

[2] H.M. Xu, C.A. Gagliardi, R.E. Tribble, A.M. Mukhamedzhanov, and N.K. Timofeyuk, Phys. Rev. Lett. 73, 2027 (1994).

[3] A.M. Mukhamedzhanov, N.K. Timofeyuk, and R.E. Tribble, Phys. Rev. C 51, 3472 (1995).

[4] A.M. Mukhamedzhanov, H.L. Clark, C.A. Gagliardi, Y.-W. Lui, L. Trache, R.E. Tribble, H.M. Xu, X.G. Zhou, V. Burjan, J. Cejpek, V. Kroha, and F. Carstoiu, Phys. Rev. C 56, 1302 (1997).

[5] C.A. Gagliardi, R.E. Tribble, A. Azhari, H.L. Clark, Y.-W. Lui, A.M. Mukhamedzhanov, A. Sattarov, L. Trache, V. Burjan, J. Cejpek, V. Kroha, S. Piskor, and J. Vincour, Phys. Rev. C 59, 1149 (1999)..

[6] L.D. Blokhintsev, V.I. Kukulin, E.V. Kuznetsova, A.A. Sakharuk, and D.A. Savin, Phys. Rev. C 48, 2390 (1993).

[7] L.D. Blokhintsev, I. Borbely, and E.I. Dolinskii, Fiz. El. Chastits At. Yadra 8, 1189 (1977) [Sov. J. Part. Nucl. 8, 485 (1977)].
[8] A.I. Baz', Ya. B. Zel'dovich, and A.M. Perelomov, Quantum Theory of Scattering and Reactions (Nauka, Moscow, 1975).

[9] J. Humblet, Phys. Rev. C 42, 1582 (1990).

[10] H. Cornille and A. Martin, Nuovo Cimento 26, 298 (1962).

[11] A.M. Lane and R.G. Thomas, Rev. Mod. Phys. 30, 257 (1958).

[12] J.M. Blatt and V.F. Weisskopf, Theoretical Nuclear Physics (Springer-Verlag, New York, 1979).

[13] C.R. Brune, Nucl. Phys. A596, 122 (1996).

[14] J. Humblet, Ann. Phys. (N.Y.) 155, 461 (1984).

[15] F.C. Barker, Nucl. Phys. A588, 693 (1995).

[16] G.R. Plattner and R.D. Viollier, Nucl. Phys. A365, 8 (1981).

[17] F.C. Barker, Phys. Lett. B 322, 17 (1994).

[18] A.B. Migdal, A.M. Perelomov, and V.S. Popov, Yad. Fiz. 14, 874 (1971) [Sov. J. Nucl. Phys. 14, 488 (1972)].

[19] E.I. Dolinskii and A.M. Mukhamedzhanov, Izv. Akad. Nauk SSSR, Ser. Fiz. 41, 2055 (1977) [Bull. Acad. Sci. USSR, Phys. Ser. 41, 55 (1977)]. 